\title{
Comparing cognitive clusters across first- and multiple-episode of psychosis
}

\author{
Geneviève SAUVÉ ${ }^{\mathrm{a}, \mathrm{b}}$, Ashok MALLA ${ }^{\mathrm{a}, \mathrm{b}, \mathrm{c}}$, Ridha JOOBER ${ }^{\mathrm{a}, \mathrm{b}, \mathrm{c}}$, Mathieu B. BRODEUR ${ }^{\mathrm{a}, \mathrm{b}}$ \\ \& Martin LEPAGE ${ }^{\mathrm{a}, \mathrm{b}, \mathrm{c}^{*}}$
}

a Douglas Mental Health University Institute, Montreal, Quebec, Canada

${ }^{\mathrm{b}}$ Department of psychiatry, McGill University, Montreal, Quebec, Canada

${ }^{\text {c }}$ Prevention and Early Intervention Program for Psychoses, Douglas Mental Health University Institute, Montreal, Quebec, Canada

* Corresponding author

Douglas Mental Health University, FBC Pavilion

6875 Blvd. LaSalle, Montreal, Québec, H4H 1R3, Canada

Phone: 1-514-761-6131 \#4393

Fax: 1-514-888-4064

Email : martin.lepage@mcgill.ca

Word count: 4,999

Abstract word count: 195

Tables : 4

Figures : 3 
Cognitive clusters across psychosis stages

Postprint - Sauvé, G., Malla, A., Joober, R., Brodeur, M. et Lepage, M. (2018). Comparing cognitive clusters across first- and multiple-episode of psychosis. Psychiatry Research, 269, 707-718. doi: 10.1016/j.psychres.2018.08.119 


\section{ABSTRACT}

Cognitive impairments in psychotic disorders (PD) present heterogeneously across patients. Between 2 and 5 clusters have been identified in previous studies with first-episode (FEP) and multiple-episodes of psychosis (MEP) patients suggesting different profiles of impairment. Past findings suggest there are differences between FEP and MEP patients regarding severity and number of affected cognitive domains. Heterogeneity of cognitive deficits in PD has perhaps hindered our understanding of their course. The present study compared non-affective FEP and MEP patients to assess whether illness chronicity could influence cognitive impairment profiles. We analyzed cognitive data, collected with the Cogstate Schizophrenia Battery, of FEP and MEP patients using cluster analysis. We compared clustering methods to obtain a more robust solution. For FEP patients, data were collected at their entry to a specialized clinic; the MEP group consisted of in- and outpatients. Results suggested cognitive heterogeneity was similar in FEP and MEP samples, although in different proportions. Three clusters were identified as the most stable solution and comprised groups of patients with either 1- no cognitive impairment (over-representation of FEP), 2- generalized deficits (over-representation of MEP), or 3intermediate impairments. These findings encourage early interventions adapted to the profile of impairment.

Keywords: cognitive impairment; schizophrenia; first-episode; enduring; heterogeneity; cluster analysis 


\section{Introduction}

Cognitive deficits, a core feature of psychotic disorders (PD), still represent an unmet therapeutic need (Gold, 2004; Heinrichs et al., 2013; Keefe et al., 2016). In 2004, the National Institute of Mental Health (NIMH) launched the Measurement and Treatment Research to Improve Cognition in Schizophrenia (MATRICS) initiative to develop treatment for cognitive deficits in schizophrenia (Green and Nuechterlein, 2004). A primary goal of this initiative was to identify distinct dimensions of cognitive impairments in PD that require clinical intervention. The workgroup identified 7 domains representing the most impaired and amenable to improvement, following treatment: speed of processing, attention/vigilance, working memory, verbal learning and memory, visual learning and memory, reasoning and problem solving, and social cognition (Green et al., 2004; Nuechterlein et al., 2004).

Impairments in these domains have also been identified in patients experiencing a first-episode of psychosis (FEP; Mesholam-Gately et al., 2009), however it remains unclear whether the severity of impairment is comparable. Using cross-sectional designs, some authors observed that FEP patients have less severe cognitive deficits than individuals who experience multiple episodes of psychosis (MEP) (Pukrop et al., 2006; Zhang et al., 2015). Yet, others have reported stable severity of cognitive impairments throughout illness progression (Corigliano et al., 2014; McCleery et al., 2014; Moritz et al., 2002; Sponheim et al., 2010). Some findings also indicate that smaller proportions of FEP patients present with cognitive deficits compared to MEP patients (Kremen et al., 2004; Lewandowski et al., 2014; Reser et al., 2015). Authors have proposed that only the more disabled patients could appear in MEP samples (Duffy et al., 2017; Iasevoli et al., 2016; McGorry et al., 2014). FEP patients with a less severe form of illness may then follow different outcome trajectories, including complete recovery and/or termination of clinical services. Illness chronicity may also increase the severity of cognitive 
impairments via lack of educational and vocational opportunities (Bergh et al., 2016; Boardman, 2011). Nonetheless, the course of cognitive deficits remains equivocal despite longitudinal investigations involving FEP patients followed up to 10 years. Bergh et al. (2016) and Rund et al. (2016) reported that the cognitive capacities of FEP patients stabilize over time, while Oie et al. (2010) observed a decline. Results of a meta-analysis including longitudinal studies with various follow-up lengths (average 12 months) also suggested equivocal findings, depicting improvement on most of the cognitive tasks analyzed, however these improvements were likely due to practice effects (Szoke et al., 2008). A more recent review of the literature on the longitudinal evolution of cognitive changes in FEP patients suggested that although deficits are present in this stage of the illness, they tend to remain stable over time (Bozikas and Andreou, 2011). The authors report on one exception though, wherein verbal memory abilities may deteriorate. Hence, although it is well established that cognition is impaired in PD, the course of impairment is still poorly understood.

One important factor to consider when refining our understanding of this issue is with respect to the highly heterogeneous nature of PD, both in terms of symptoms and underlying cognitive deficits (Seaton et al., 2001). Previous results using cluster analyses are unclear; solutions ranging from 2 to 5 clusters (i.e., distinct cognitive profiles) have been reported in the literature. The nature of impairments across clusters also differ within studies. Identified clusters are comprised of patients with 1- severe and generalized cognitive impairments (between 15-25\%), 2- normal cognitive functioning (between 20-40\%), or specific deficits in 3- visuospatial processing and memory, 4- executive functions, and/or 5- verbal memory (Gilbert et al., 2014; Goldstein et al., 1998; Hill et al., 2002; Kremen et al., 2004; Lewandowski et al., 2017; Lewandowski et al., 2014). To our knowledge, three studies investigated a similar question in the FEP population, and all reported divergent findings (Ilonen et al., 2004; Reser et al., 2015; Uren et al., 2017). Ilonen et al. (2004) identified 3 clusters comprising FEP patients with 


\section{Cognitive clusters across psychosis stages}

either 1- normal intellectual and memory function, 2- normal IQ accompanied by moderate memory impairments, or 3- mild mental retardation combined with severe memory deficits. Similarly, Uren et al. (2017) identified 3 clusters of FEP patients and healthy control participants with either 1generalized impairments, 2- moderate deficits, or 3- intact performance. Conversely, Reser et al. (2015) identified 4 clusters of FEP patients presenting 1- global cognitive impairments, 2- normal cognitive capacities, 3- visual memory deficits, or 4- attentional and working memory impairments. Importantly, these results may have been influenced in part by the use of different cognitive test batteries.

The use of various testing protocols across studies using cluster analysis has limited our understanding of the differences between FEP and MEP patients regarding the heterogeneity, severity, and cognitive profile patterns. Not all prior studies included control participants, and most used different clustering algorithms, cognitive tests, administration procedures, and scoring and standardization techniques. Further, some studies included both affective and non-affective psychosis diagnoses, which can be problematic as patients with these diagnoses may present different profiles of cognitive impairment severity (Bora, 2016). To our knowledge, no study has included different groups based on illness stage, and few have compared solutions obtained through different clustering algorithms.

In the current study, we performed cluster analyses on a sample comprising both FEP and MEP patients to investigate whether cognitive heterogeneity is comparable across the psychosis continuum. Given the general trend of previous findings, we hypothesized that FEP and MEP patients would respectively present with more heterogeneous and homogeneous profiles of cognitive impairments. More precisely, we anticipated obtaining a final solution containing at least 3 clusters (namely 1normal performance, 2- generally impaired, and 3- specific impairments). While we expected MEP 
patients to be over-represented in the second cluster, we also predicted finding comparable proportions of FEP and MEP patients in the other two clusters. Healthy control participants were included in our analyses to verify whether they would aggregate with patients in our predicted 'normal performance' cluster.

To achieve our objective, cognitive data were collected from FEP and MEP patients. One inclusion criterion that cut across both groups was the requirement to have a diagnosis of non-affective psychosis. It follows that individuals with bipolar disorder or major depressive disorder with psychotic features were excluded from either patient groups. FEP patients were recruited through a psychosis clinic specialized in early intervention. To be admitted, individuals must have received less than one month of antipsychotic treatment in their lifetime indicating that it was the first time they were receiving services for their psychotic symptoms. MEP patients were recruited via in- and outpatient services and had been receiving psychiatric treatment for psychotic illness for a minimum of 4 years indicating that they had experienced more than one psychotic episode. Patients from each group were matched to healthy controls on age, sex, and education. All patients' cognitive performance scores were subsequently standardized to that of healthy control participants.

\section{Methods}

\subsection{Participants}

\section{$\underline{2.1 .1 ~ F E P ~ a n d ~ F E P-C o n t r o l ~ g r o u p s ~}$}

FEP participants were recruited through the PEPP-Montreal program (see Iyer et al., 2015), which specializes in early psychosis intervention and is located at the Douglas Mental Health University Institute (DMHUI) in Montreal, Canada. Being the only FEP service in its catchment area of about 400,000 inhabitants, this specialized clinic serves a nearly complete incidence sample (Iyer et al., 
2015). For the current sample, $49 \%$ of FEP patients were referred to PEPP-Montreal from the emergency department, $16 \%$ from inpatient units, $8 \%$ were self-referred or referred by a family member, 7\% were referred from community services, 7\% from an affiliated ultra-high risk clinic, and the remaining $10 \%$ from school, community psychiatrist, and other sources. The program admits individuals aged between 14 and 35 presenting with affective and non-affective psychosis. All are invited to participate in a longitudinal study investigating cognition and clinical outcomes in FEP. All participants included in the present study provided informed written consent in a manner approved by the local ethics committee. Participants had not taken antipsychotic medication for more than one month prior to entry. Diagnoses were established using the Structured Clinical Interview for DSM-IV (SCID DSM-IV; Biometrics Research, New York, USA) (First et al., 1998) and via consensus of two senior psychiatrists (A.M \& R. J.).

Eighty FEP patients were included in the current analyses. Exclusion criteria were 1- diagnoses of affective psychosis or substance-induced psychosis, 2- a score below 70 on the IQ measurement, and 3- not having completed the cognitive testing. For comparison purposes, FEP patients with affective psychosis were not included as our MEP group consisted only of non-affective psychosis. Given the early stage and mission of the PEPP-Montreal clinic, diagnoses are classified as schizophreniaspectrum disorder or affective psychosis. Therefore, only patients with the former diagnosis were included in the current analyses. Intelligence decline, defined as a decrease of 10 points or more between the current IQ score and the premorbid IQ estimate (Ohi et al., 2017), was not an exclusion criterion and was observed in 12 FEP patients. Similar results were obtained when we specifically analyzed verbal intelligence decline. An estimation of premorbid IQ was measured only in FEP patients as part of their evaluation upon their entry in the clinic. 
Cognitive clusters across psychosis stages

As part of the same longitudinal study, 55 healthy controls were recruited from within the same catchment area and completed the same cognitive assessment. No control participants reported a personal or family history of psychiatric illness, history of concussion, head trauma, or brain lesions. These individuals formed the FEP-Control group and were matched to FEP patients on age, sex and education.

\subsubsection{MEP and MEP-Control groups}

MEP participants $(n=121)$ were recruited as part of a larger cross-sectional study conducted at the DMHUI aimed at investigating the psychological and neuronal determinants of insight in schizophrenia (see Emami et al., 2016). In- and outpatients aged between 18 and 50 were recruited from local mental health resources and had been receiving psychiatric treatment for a minimum of four years. Despite the relative young age of some patients, those included in this study were all multiepisode cases as confirmed by review of their medical chart. Diagnoses of non-affective psychoses were established using the SCID DSM-IV and medical chart review. The proportions of the diagnoses are as follow: $n=98$ for schizophrenia, $n=31$ for schizoaffective, $n=1$ for psychosis not otherwise specified, and $n=1$ delusion disorder. Exclusion criteria were the same plus 1 - family or personal history of neurological condition, and 2- substance dependence diagnosis within the last three months. As the larger study included a Magnetic Resonance Imaging (MRI) portion, participants were also excluded if they had any contraindication for MRI scanning. The study was approved by the DMHUI's ethics committee and all participants signed a written consent form.

Seventy healthy control participants were recruited through local media and matched to MEP patients on age, sex, and education. Exclusion criteria were the same as those for FEP-Control individuals. Due to the MRI portion, MEP-Controls additionally had no contraindication for scanning. 


\subsection{Assessments}

\subsubsection{Sociodemographic and clinical measures}

All data collection was performed by trained research assistants or students who were not involved in patients' treatment program. At the time of cognitive testing, all participants provided information regarding their age, sex, and years of education. MEP patients also provided additional written consent to allow the research team consult their medical chart. This was conducted to confirm diagnosis and to evaluate duration of illness, defined as the period between the year of first hospitalization or reported psychotic symptoms and the date of participation in the study.

Symptoms were assessed for all patients using the Scale for the Assessment of Positive Symptoms (SAPS) and the Scale for the Assessment of Negative Symptoms (SANS). Depression and anxiety were assessed using the Calgary Depression Scale for Schizophrenia (Addington et al., 1990) and the Hamilton Anxiety Rating Scale (Hamilton, 1959), respectively. Inter-rater reliability scores for the SAPS-SANS interview were 0.72 for FEP patients and 0.83 for MEP patients.

\section{$\underline{\text { 2.2.2 Cognitive measures }}$}

All participants completed the computer-based CogState Schizophrenia Battery (Cogstate, New York, USA), which was developed to measure the cognitive dimensions recommended by the MATRICS workgroup (Lees et al., 2015). Therefore, speed of processing was measured with the 'Detection task' and the 'Groton maze chase test'; attention/vigilance was evaluated with the 'Identification task'; working memory used the 'One-back' and 'Two-back' memory tests; verbal learning and memory was estimated with the 'International shopping list' and its delayed recall; reasoning and problem solving were measured with the 'Groton maze learning test' and the 'Set- 
shifting test'; visual learning and memory was evaluated with the 'One card learning task, the 'Continuous paired associated learning task' and a delayed recall of the 'Groton maze learning test'; and finally social cognition was estimated using the 'Social-Emotional Cognition task'. The battery takes 50 minutes on average to administer and has been shown to be valid and reliable in both FEP and MEP samples (Benoit et al., 2015; Pietrzak et al., 2009). An interesting advantage of the Cogstate battery is that all these tests avoid relying on fine motor skills (Benoit et al., 2015). Moreover, they make minimal language requirement for the completion of most tasks, except those measuring verbal learning and memory, which reduces cultural confounds (Benoit et al., 2015; Lees et al., 2015).

Current full-scale IQ of all participants was measured using the Wechsler Abbreviated Scale of Intelligence (WASI; Pearson, San Antonio, USA). The North-American Adult Reading Test (NAART) was used to estimate premorbid global, verbal and performance IQ in FEP patients only (Blair and Spreen, 1989). The English version of the NAART has shown good psychometric properties in nonclinical samples (Uttl, 2002) and has been successfully used in patients with a psychotic disorder (Baitz et al., 2012; Fiszdon et al., 2006; Lee et al., 2012; Wilder-Willis et al., 2002). Current IQ evaluation was often completed independently of the CogState administration to avoid mental fatigue.

As FEP patients were part of a longitudinal study, their clinical evaluation was performed at different predetermined time-points, namely at $1,2,3,6,9,12$, and 18 months following program entry. Baseline cognitive evaluations were conducted at maximum 3 months after program entry once patients were clinically stable. At the time of cognitive testing, the vast majority of FEP patients were seen on an outpatient basis. Clinical scales scores used in the current analyses were selected according to the time-point closest to cognitive testing. 


\subsection{Statistical analysis}

\subsubsection{Description of the data}

One-way ANOVA were used to analyze sociodemographic and clinical data. Kruskall-Wallis tests followed-up by Mann-Whitney U tests were used for non-parametric data or when parametric assumptions were not met. For all analyses, the alpha level was set at .05 and the Bonferroni method was used to correct for multiple comparisons (the maximum number of comparisons was 3; $\alpha=$ $0.05 / 3)$.

\subsubsection{Identifying clusters}

\section{Standardization and outlier detection}

Composite scores for each MATRICS dimension were z-transformed according to the mean and standard deviation of a subsample of healthy control participants (CTL-FEP $n=67$; CTL-MEP $n=52$ ) for each patient group; procedure is detailed in Benoit et al. (2015). Healthy control participants were matched to patients on age, sex and years of education. They were recruited from the community to participate in the same studies in which the patients took part. Hence, patients with negative scores exhibit poorer performance as compared to the respective healthy control group. A deficit was defined as a z-score below -1, reflecting a performance of 1 standard deviation below the healthy controls average. Univariate and multivariate outliers were identified using the technique developed by Caroni and Prescott (1992) and detected with the Excel Macro provided in Achim (2012). Five MEP participants were identified as such on the cognitive variables and thus were excluded from the cluster analyses because these techniques are sensitive to outliers.

Hierarchical cluster analysis 
Cognitive clusters across psychosis stages

Hierarchical clustering analyses uses an iterative-additive procedure wherein similar cases are aggregated together forming homogeneous groups. We ran the analysis on our entire sample (all groups combined) using different methods (1- Single linkage, 2- Complete linkage, 3- Average linkage) to compare solutions, and the squared Euclidean distance was chosen as the interval measure (Cross, 2013; Everitt et al., 2011). As a guide to the cutting point of dendrograms, we followed the procedure described in Yim and Ramdeen (2015), which proposes to examine the agglomeration schedule to find the first large difference between two consecutive clusters. The final selection of the number of clusters in the solution was made after careful examination of dendrograms.

\subsubsection{Optimization and validation of cluster solutions}

K-means cluster analysis

After reaching the final cluster solution for each hierarchical method, K-means cluster analyses were performed on the whole sample (all groups combined) to optimize solutions. Because this type of analysis requires the number of clusters to be decided a priori, it can be used as an optimization method once the number of clusters have been identified using other methods, such as hierarchical cluster analysis (Everitt et al., 2011). By utilizing an additive procedure, hierarchical methods represent a strict iteration process in that once cases are included in a cluster, they cannot be taken out. K-means analysis allows cases to move from one cluster to another across iterative stages and re-calculates clusters centers at each iteration stage to obtain a final optimal solution wherein clusters are most compact and distant from each other (Everitt et al., 2011).

K-means cluster analyses were thus run with 2 and 3 clusters according to results obtained in the complete and average linkage methods used in the hierarchical analysis portion. We used the 'iterate and classify' method, and the convergence criterion was set to 0 . The maximum number of 
iterations was set so that the resulting solution would reach the convergence criterion. Hierarchical and K-means cluster analyses were conducted using SPSS.

\section{Adjusted Rand Index (ARI)}

The ARI (Hubert and Arabie, 1985) compares clustering solutions and was calculated using the Mclust package (Fraley and Raftery, 2002; Fraley et al., 2012) in Rstudio (1.0.136) (RStudio Team, 2015). We used the ARI to validate our optimized solutions by statistically comparing the classifications obtained through the hierarchical and K-means analyses (e.g., hierarchical analysis 3cluster solution vs K-means analysis 3-cluster solution) (Lee et al., 2017). The ARI is represented by a value between -1 (suggesting no agreement between the two solutions compared) to +1 (suggesting perfect agreement). A general guideline for the interpretation of ARI scores is that higher values indicate better agreement between the two clustering solutions compared.

\section{Discriminant function analysis (DFA)}

DFA can be used in conjunction with cluster analysis to evaluate the performance of clustering solutions because it allows one to verify whether a set of variables can correctly classify cases across predefined groups. An important assumption of linear DFA is homogeneity of variance-covariance matrices across groups (or clusters), which can be verified using Box's test (Tabachnick and Fidell, 2013). Because our matrices were heterogeneous, we opted to conduct a quadratic DFA (qDFA) as it does not require homogeneity of covariance matrices. This analysis was conducted on the whole sample with the MASS package (Venables and Ripley, 2002) in Rstudio (1.0.136) (RStudio Team, 2015). We used the jackknife reclassification approach as it allows one to use the same data for both the cluster and DFA analyses (Efron, 1982), and it reduces bias of model cross-validation (Cross, 2013). 


\subsubsection{Comparing clinical profiles across clusters}

MANOVA were conducted on IQ, sociodemographic (years of education, age) and clinical

measures (anxiety, depression, positive and negative symptoms, age of psychosis onset, and chlorpromazine equivalents) of patients and healthy controls (where applicable) across clusters. Kruskall-Wallis tests (followed-up by Mann-Whitney U tests) and Independent-samples t-tests were used for non-parametric variables (e.g., sex) or when MANOVAs assumptions were not met. The significance threshold was corrected for multiple comparisons using the Bonferroni method.

\subsubsection{Comparing heterogeneity in FEP and MEP samples within clusters}

FEP and MEP patients were compared within cluster on cognitive, sociodemographic, and clinical variables. For cognitive variables, one MANOVA with 2 between-subject factors (1- Cluster membership, 2- Clinical group) and 1 within-subject factor with 7 levels (MATRICS domains) was conducted. For the sociodemographic and clinical variables, another MANOVA was used with the same between- and within-subject factors, but the latter had 8 levels (medication, age, full IQ, verbal IQ, performance IQ, years of education, global score for hallucinations and global score for alogia). MANCOVAs were additionally run with chlorpromazine equivalents as a covariate. Pearson Chisquare tests were conducted to statistically compare proportions of FEP vs MEP patients within clusters obtained in optimized solutions on the statistically significant variables identified in the MANOVAs.

\subsubsection{Predicting cluster membership from stage of illness}

Hierarchical multiple regression analyses were conducted to verify whether stage of illness predicted cluster membership, while controlling for relevant clinical variables. The enter method was 
used and covariates were selected based on whether there was a significant difference across clusters. Control variables were entered in the first step of a hierarchical regression, while clinical group (FEP vs MEP) was entered in the second step.

\subsubsection{Additional analyses}

We ran the same statistical protocol on a subsample excluding the control groups to verify the stability and interpretability of our results. Because cluster analysis depends heavily on the dataset used, finding similar cognitive clusters in analyses that do and do not include control participants will further validate our results. Results of additional analyses are available in online Supplementary Material.

\section{Results}

\subsection{Description of data}

Sociodemographic and clinical characteristics are presented in Table 1. Mean z-scores by MATRICS domain per clinical group are presented in Supplementary Material (Fig S2).

\subsection{Cluster analysis}

\subsubsection{Hierarchical agglomerative method}

The single-linkage method resulted in a no-cluster solution according to close examination of the dendrogram and agglomeration schedule (dendograms in Figure 1; agglomeration schedule in Figure S1). The complete-linkage method yielded a 3-cluster solution and the average-linkage one reached a 2-cluster solution.

\subsubsection{K-means analysis (optimization)}


Cognitive clusters across psychosis stages

K-means cluster analyses was completed with $k=2$ and $k=3$ to optimize hierarchical solutions, as suggested in Everitt et al. (2011). Figure 2A-B shows mean performance of participants included in each cluster on each MATRICS domain. In the $k=2$ solution, cluster 1 was labelled 'No Impairment' $(N I)$ and comprised individuals with z-scores above -1 on all domains; cluster 2 was labelled 'Generally Impaired' $(G I)$ and comprised individuals with z-scores below - 1 for every domain. In the $k$ = 3 solution, cluster 1 was labelled 'No Impairment' $(N I)$, cluster 2 was labelled 'Generally Impaired' (GI), and cluster 3 was labelled 'Intermediately Impaired' (II). A deficit being defined as a z-score below -1 , the intermediately impaired participants showed deficits in the following domains: verbal learning and memory; working memory; and reasoning and problem solving. Figure 3A-B illustrates clinical group distributions (FEP, MEP, Ctl-FEP, Ctl-MEP) for each cluster.

\subsubsection{Validation}

The ARI comparing the 3-cluster solutions obtained through hierarchical analysis vs. K-means analysis was 0.41. The index for the 2-cluster solutions comparison was 0.14, suggesting the optimization procedure provided a more satisfactory and stable solution for $k=3$ than for $k=2$.

The qDFAs yielded correct classification rates of 91.4\% for the K-means cluster solution with $k=3$ and $65.0 \%$ with $k=2$, suggesting the 3-cluster solution performed better than the 2-cluster solution.

\subsubsection{Comparing clinical profiles across clusters}

Clusters were compared on multiple variables: full IQ, global scores from SAPS and SANS respectively for positive and negative (excluding attention) symptoms, age, sex, years of education, anxiety, and depression. For solution $k=3$, Kruskall-Wallis tests showed significant differences between clusters on IQ, global scores of hallucination and alogia, age, and years of education. Results 
were similar for solution $k=2$. Participants in the $N I$ cluster had higher IQ, more years of education, were younger, and had less severe hallucination and alogia ratings. Individuals in the GI cluster had accordingly lower IQ, fewer years of education, and were older. They had higher level of alogia and hallucinations ratings ranging between those of the NI and II clusters. Means and standard deviations of sociodemographic and clinical variables for each cluster of solutions $k=3$ and $k=2$ are presented in Table 2. Comparisons between FEP and MEP subsamples within each cluster of the solution $k=3$ for the sociodemographic and clinical variables are presented in Table 3. Notably, FEP patients were unsurprisingly younger and had a shorter duration of illness within all clusters.

\subsubsection{Comparing cognitive heterogeneity in FEP and MEP samples within cluster}

There was no significant difference between FEP and MEP samples on cognitive performance within clusters $(F(14,378)=1.69, \mathrm{p}>0.05)$ (Table 4). The same results were obtained for the MANCOVA controlling for chlorpromazine equivalents. Although the omnibus analysis reported a significant Clinical group by Cluster membership interaction $(F(12,374)=1.92, p<0.05)$, post hoc comparisons on all MATRICS domains failed to show significant difference between clinical groups in each cluster (all $p>0.05$ ). For the $k=3$ solution, Chi-square tests revealed a significantly higher

proportion of FEP in the NI cluster $\left(\chi^{2}(1)=17.5, p<0.001\right)$. MEP patients were represented in significantly larger proportions in the $G I$ cluster $\left(\chi^{2}(1)=8.25, p<0.005\right)$. For the $k=2$ solution, proportions of FEP and MEP significantly differed in both clusters (all $\chi^{2}(1)>25.2$, all $p<0.001$ ).

\section{$\underline{\text { 3.2.6 Predicting cluster membership from stage of illness }}$}

Stage of illness significantly predicted cluster membership for both the $k=3(\beta=0.14, \mathrm{~b}=$ 0.25 , SE $\mathrm{b}=0.14$, adjusted $\left.\mathrm{R}^{2}=0.12, p<0.001\right)$ and $k=2$ solutions $(\beta=0.29, \mathrm{~b}=0.30, \mathrm{SE} \mathrm{b}=0.07$, 
adjusted $\left.\mathrm{R}^{2}=.17, p<0.001\right)$. More specifically, stage of illness was among the top predictors and accounted for $12 \%$ and $17 \%$ of cluster membership’s variance. Results are presented in Table 5.

Only results of the 3-cluster optimized solution will be discussed as this solution represents the most stable and interpretable one; it also replicates previous findings (Gilbert et al., 2014; Ilonen et al., 2004; Uren et al., 2017).

\section{Discussion}

\subsection{Summary of findings}

The present study investigated whether cognitive heterogeneity was comparable in FEP and MEP patients. We conducted cluster analyses on cognitive data of FEP and MEP patients and their matched healthy control participants using the CogState battery. We obtained a final 3-cluster solution characterizing distinct profiles (1- No impairment, NI; 2- General impairment, GI; and 3- Intermediate impairment, II), which was in line with our hypothesis. Similar solutions were obtained in additional analyses including only patients (i.e., no healthy control participants), for which results are available in Supplementary Material (Figures S3-S6). In addition, our results suggest there was a significantly higher proportion of FEP in the $N I$ cluster as well as a larger representation of MEP patients in the GI cluster. Further, we observed no difference between FEP and MEP patients within clusters on cognitive performance, indicating 1- some FEP patients (although fewer) exhibit cognitive profiles as impaired and widespread as MEP patients, and 2- some MEP patients (although fewer) display no impairment, similar to FEP patients. Interestingly, we also observed that the GI cluster had more severe levels of alogia than the other two clusters, while not significantly differing from the II cluster on antipsychotic dosage and severity of hallucinations. The potential impact of these variables on the observed cognitive heterogeneity is discussed. 


\subsection{Influence of clinical and sociodemographic variables on cognitive heterogeneity}

Along with stage of illness, other different clinical and sociodemographic variables may concurrently have an influence on cognitive heterogeneity. Given the complex relationship between cognitive functioning and antipsychotic medications, an interesting finding from our study concerns the statistically similar antipsychotic dosages between 1- FEP and MEP patients within clusters, and 2patients in the GI and II clusters. While negative effects on speed of processing and verbal learning and memory have been observed for higher daily doses of antipsychotics in MEP patients (Rehse et al., 2016), procognitive effects have also been observed in other studies for some other types of antipsychotic medications. A recent meta-analysis reported that quetiapine and olanzapine were associated with greater improvement in overall cognition in both FEP and MEP samples as compared to amisulpride and haloperidol (Desamericq et al., 2014). In more exploratory work, Lepage et al. (2015) found specific improvements in verbal memory following one year of treatment with aripiprazole in a sample of FEP patients. Similar results were reported in another study investigating a large mixed FEP and MEP sample (Bervoets et al., 2012). Nevertheless, patients who showed the lowest levels of cognitive impairments in our sample also had the lowest antipsychotic dosages, suggesting that other factors may impact cognitive heterogeneity in FEP and MEP patients.

To that effect, one potential explanation for the higher dosages of antipsychotic medications observed in the GI cluster comes from genetic studies. In addition to being more medicated and showing more severe positive symptoms, GI patients also show lower IQ levels. Findings from a metaanalysis suggest lower IQ is associated with higher risk of developing a PD (Khandaker et al., 2011). It has been hypothesized that the genetic liability on risk for PD is accentuated in individuals with lower IQ levels (Kendler et al., 2015). It is therefore possible that patients in our GI cluster represent those 
who were at higher risk of developing more severe positive symptoms, thus requiring more antipsychotic medication.

Another factor possibly influencing the observed cognitive heterogeneity has to do with the interfering effect that psychotic symptoms may exert on cognitive performance. Our results suggested hallucinations were more severe in the $G I$ and $I I$ clusters, and that alogia was more important in the GI cluster. It is possible that auditory hallucinations and symptoms characterizing alogia, such as blocking and increased latency of response, interfere with cognitive strategies used during cognitive testing, such as mental verbalization. This strategy is taught in some cognitive remediation therapies to enhance focus and accuracy, and requires one to mentally describe their thought process and actions during task completion (Barlati et al., 2013). These symptoms could likely impede the use of mental strategies, thus resulting in decreased performance. A similar situation could arise when using more visual strategies, such as visual scanning, in the context of visual hallucinations.

In addition to the previous clinical variables, cognitive heterogeneity also seemed to be influenced by more sociodemographic indices, namely IQ, age and years of education. One finding from our study is that IQ levels and years of education gradually decreased across cluster $(N I>I I>$ GI). A similar result was obtained for age in that individuals in the $N I$ cluster were younger than those in the II and GI clusters. Similar clusters have been observed in other research applying the same type of methodology to IQ scores (Seaton et al., 1999; Weickert et al., 2000). The identification of a cluster comprising patients with an intellectual decline has led Weickert et al. (2000) to suggest that cognitive and intellectual deficits may emerge from different neurodevelopmental trajectories. Furthermore, Seaton et al. (1999) proposed that the relative independence of cognitive clusters from symptom severity could reflect stable trait characteristics. Such neurodevelopmental abnormalities may translate 
Cognitive clusters across psychosis stages

into educational achievement potentially explaining the difference between clusters on this variable. Similarly, these neurodevelopmental anomalies may not be observable via cognitive testing in younger patients, explaining the age difference across clusters. However, these hypotheses need to be empirically tested. Taken together, the findings reported in this study seem to suggest that patients' cognitive profile may be determined early in the course of illness and influenced by several factors.

\subsection{Interventions}

Our results have important implications for the timing and content of cognitive interventions given that larger proportions of MEP patients were found in the GI cluster, and more FEP patients were included in the NI cluster. Cognitive remediation therapy (CRT) may be beneficial at any stage; yet, in the context of CRT, inverse correlations between cognitive improvement and illness chronicity have been reported, indicating that early intervention may be offered preventively (Bowie et al., 2014). Moreover, being younger in age has been reported as a significant predictor of response to CRT (Lindenmayer et al., 2017). It has also been suggested that cognitive impairments could interfere with the ability to benefit from psychosocial treatment, emphasizing the value of early cognitive remediation intervention (Ilonen et al., 2004).

Despite the apparent advantages of early cognitive intervention, it may also be beneficial to tailor the content of therapy to each patient's cognitive impairments, rather than to stage of illness only, since cognitive heterogeneity seems to be present in both FEP and MEP patients (Lewandowski et al., 2014). Moreover, group interventions could gather together patients with similar profiles of cognitive impairments. Hence, patients with selective impairments may benefit from shorter, more focused interventions, potentially improving concurrent treatment adherence (Twamley et al., 2011). Additionally, combining cognitive remediation with more intensive approaches such as employment 
support programs (Chan et al., 2015) for patients with a general impairment profile may also be an effective avenue (Reser et al., 2015). Interestingly, recent preliminary findings by Nuechterlein and collaborators showed that combining CRT with a program of aerobic exercises enhanced cognition and functioning gains beyond the effects of CRT alone (Nuechterlein et al., 2018; Nuechterlein et al., 2016). The authors also indicated that the effects of this therapeutic combination translated into positive brain changes like increased prefrontal cortical thickness and functional connectivity. Overall, it seems that CRT may benefit from being adapted to patients’ cognitive profiles.

\subsection{Limitations}

The current study has some limitations that need to be acknowledged. First, our sample size was limited. Second, given that cluster analysis is highly influenced by the inputted datasets, variables, the clustering algorithm and the criteria used to determine the number of clusters, we cannot state that our results reflect fundamental cognitive subtypes (Everitt et al., 2011). Rather, we propose that cognitive heterogeneity is similar, although in different proportions, in FEP and MEP patients. We nevertheless tried to circumvent the limitations of cluster analysis by comparing different linkage methods and by running multiple internal and external validation analyses (Everitt et al., 2011). Comparing linkage methods is encouraged since obtaining converging and similar solutions provide a basis to determine the reliability of such results. Conversely, obtaining very discrepant solutions could indicate that there is no meaningful underlying structure or subgroups within the dataset (Everitt et al., 2011). As Goldstein (2013) suggests, ultimately, researchers should choose the most interpretable, robust and parsimonious solutions that can be derived from their dataset. Third, the cross-sectional nature of our study does not allow for any claims regarding the course of patients’ cognitive performance. Ideally, a longitudinal follow-up of FEP patients would reveal the post-treatment factors found to influence cognitive changes occurring after treatment of a first episode. We believe however that by pinpointing 
different cognitive clusters found in both FEP and MEP patients, our findings are at least partially complementary to longitudinal studies. Fourth, computerized cognitive testing may have been more difficult for older patients, which could have selectively affected those most likely included in the MEP group. It would be interesting to replicate our findings with pen and paper cognitive evaluations. Fifth, patient groups likely differed in the cumulative exposure to antipsychotics, but we could not control for this potential confound as such data was not readily available from the patient's chart. Finally, it could be argued that our findings may be explained by the fact that MEP patients typically have a more severe form of illness and thus present with more generalized cognitive impairments. While being a completely valid proposition, it should not be discounted that illness chronicity may be accompanied by a lack of opportunity to maintain adequate levels of cognitive capacity, for instance, via the daily challenging of different cognitive functions through work, school, etc. This impoverished environment may subsequently result in a stage of illness comprising more homogeneous profiles of generalized cognitive impairments.

\subsection{Conclusion}

Our results suggest that the cognitive heterogeneity in FEP and MEP samples is comparative. Yet, multiplicity of psychotic episodes may have a detrimental impact on cognition, as we observed that a larger proportion of MEP patients present with the generally impaired profile. We thus argued it could be beneficial to implement early cognitive interventions adapted to impairment profiles. Combined with psychosocial interventions such as evidence-based employment support programs like Individual Placement and Support (IPS), cognitive deterioration may then be better prevented through direct and indirect training of cognitive skills. While CRT may offer direct training, opportunities for continuing school or work may represent indirect opportunities to further train such abilities. Finally, the contradicting results previously reported regarding the course of cognitive deficits in PD might 
have been influenced by the inclusion of patients with differing impairment profiles. It would be interesting in the future to investigate whether cluster membership is amenable to change (e.g., switching from the generally impaired cluster to the intermediately impaired) longitudinally or following treatment (Heinrichs et al., 1997).

\section{Acknowledgments}

The authors are grateful to the staff of the Martin Lepage Laboratory and PEPP-Montreal for helping with recruitment and data collection. We also wish to thank all participants. The study was supported by operating grants from the Canadian Institutes of Health Research (CIHR; \#68961, \#106634), the Sackler Foundation and Bristol-Myers Squibb to M.L./A.M. as well as an investigator-initiated study grant from the Otsuka/Lundbeck Alliance (\#20135257) to M.L. Salary awards include: Fonds de Recherche du Québec - Santé (FRQ-S) to G.S, M.B.B. and R.J.; James McGill Professorship and FRQ-S Research Chair to M.L.; Canada Research Chairs Program to A. M. Author ML reports having received financial assistance/compensation for research and educational events from Otsuka/Lundbeck Alliance, all outside the submitted work. Author RJ reports to be a speaker and/or consulting committee member for Pfizer, Janssen, BMS, Sunovian, Myelin, Otsuka, Lundbeck, shire and Perdue, and to have received grants from Janssen, BMS, Otsuka, Lundbeck, Astra Zeneca and HLS, and to have royalties from Henry Stewart talks, all outside the submitted work. Author AM reports having received grants, fees, or honoraria from BMS, Lundbeck, and Otsuka, all outside the submitted work. Authors GS and MBB report no conflict of interest.

\section{REFERENCES}

Achim, A., 2012. Detecting outliers in multivariate data while controlling false alarm rate. Tutor Quant Methods Psychol 8 (2), 108-121. 
Addington, D., Addington, J., Schissel, B., 1990. A depression rating scale for schizophrenics. Schizophr Res 3 (4), 247-251.

Baitz, H.A., Thornton, A.E., Procyshyn, R.M., Smith, G.N., MacEwan, G.W., Kopala, L.C., et al., 2012. Antipsychotic medications: linking receptor antagonism to neuropsychological functioning in first episode psychosis. J Int Neuropsychol Soc 18 (4), 717-727.

Barlati, S., Deste, G., De Peri, L., Ariu, C., Vita, A., 2013. Cognitive remediation in schizophrenia: current status and future perspectives. Schizophr Res Treatment 2013, 156084.

Benoit, A., Malla, A.K., Iyer, S.N., Joober, R., Bherer, L., Lepage, M., 2015. Cognitive deficits characterization using the CogStage Research Battery in first-episode psychosis patients. Schizophrenia Research: Cognition 2, 140-145.

Bergh, S., Hjorthoj, C., Sorensen, H.J., Fagerlund, B., Austin, S., Secher, R.G., et al., 2016. Predictors and longitudinal course of cognitive functioning in schizophrenia spectrum disorders, 10years after baseline: The OPUS study. Schizophr Res 175 (1-3), 57-63.

Bervoets, C., Morrens, M., Vansteelandt, K., Kok, F., de Patoul, A., Halkin, V., et al., 2012. Effect of aripiprazole on verbal memory and fluency in schizophrenic patients : results from the ESCAPE study. CNS Drugs 26 (11), 975-982.

Blair, J., Spreen, O., 1989. Predicting premorbid IQ: a revision of the national adult reading test. Clin Neuropsychol 3 (2), 129-136.

Boardman, J., 2011. Social exclusion and mental health - how people with mental health problems are disadvantaged: an overview. Mental Health and Social Inclusion 15 (3), 112-121.

Bora, E., 2016. Differences in cognitive impairment between schizophrenia and bipolar disorder: Considering the role of heterogeneity. Psychiatry Clin Neurosci 70 (10), 424-433.

Bowie, C.R., Grossman, M., Gupta, M., Oyewumi, L.K., Harvey, P.D., 2014. Cognitive remediation in schizophrenia: efficacy and effectiveness in patients with early versus long-term course of illness. Early Interv Psychiatry 8 (1), 32-38.

Bozikas, V.P., Andreou, C., 2011. Longitudinal studies of cognition in first episode psychosis: a systematic review of the literature. Aust N Z J Psychiatry 45 (2), 93-108.

Caroni, C., Prescott, P., 1992. Sequential application of Wilk's multivariate outlier test. Applied statistics 41, 355-364.

Chan, J.Y., Hirai, H.W., Tsoi, K.K., 2015. Can computer-assisted cognitive remediation improve employment and productivity outcomes of patients with severe mental illness? A meta-analysis of prospective controlled trials. J Psychiatr Res 68, 293-300.

Corigliano, V., De Carolis, A., Trovini, G., Dehning, J., Di Pietro, S., Curto, M., et al., 2014. Neurocognition in schizophrenia: from prodrome to multi-episode illness. Psychiatry Res 220 (1-2), 129-134. 
Cross, C.L., 2013. Statistical and methodological considerations when using cluster analysis in neuropsychological research, in: Allen, D.N., Goldstein, G. (Eds.), Cluster Analysis in Neuropsychological Research. Springer, NY, USA, pp. 13-36.

Desamericq, G., Schurhoff, F., Meary, A., Szoke, A., Macquin-Mavier, I., Bachoud-Levi, A.C., et al., 2014. Long-term neurocognitive effects of antipsychotics in schizophrenia: a network metaanalysis. Eur J Clin Pharmacol 70 (2), 127-134.

Duffy, A., Malhi, G.S., Grof, P., 2017. Do the trajectories of bipolar disorder and schizophrenia follow a universal staging model? Can J Psychiatry 62 (2), 115-122.

Efron, B., 1982. The Jackknife, the Bootstrap and Other Resampling Plans. Society for Industrial and Applied Mathematics, Philadelphia, PA.

Emami, S., Guimond, S., Mallar Chakravarty, M., Lepage, M., 2016. Cortical thickness and low insight into symptoms in enduring schizophrenia. Schizophr Res 170 (1), 66-72.

Everitt, B.S., Landau, S., Leese, M., Stahl, D., 2011. Cluster Analysis. John Wiley \& Sons, Ltd, UK.

First, M.B., Spitzer, R.L., Gibbon, M., Williams, J.B.W., 1998. Structured Clinical Interview for DSM-IV Axis I Disorderes, Patient Edition (SCID-P \& SCID-NP), Version 2. New York Psychiatric Institute, Biometrics Research, New York.

Fiszdon, J.M., McClough, J.F., Silverstein, S.M., Bell, M.D., Jaramillo, J.R., Smith, T.E., 2006. Learning potential as a predictor of readiness for psychosocial rehabilitation in schizophrenia. Psychiatry Res 143 (2-3), 159-166.

Fraley, C., Raftery, A., 2002. Model-based clustering, discriminant analysis and density estimation. Journal of American Statistical Association 97, 611-631.

Fraley, C., Raftery, A., Murphy, B., Scrucca, L., 2012. mclust Version 4 for R: Normal mixture modeling for model-based clustering, classification and density estimation Technical Report No. 597, Department of statistics, University of Washington.

Gilbert, E., Merette, C., Jomphe, V., Emond, C., Rouleau, N., Bouchard, R.H., et al., 2014. Cluster analysis of cognitive deficits may mark heterogeneity in schizophrenia in terms of outcome and response to treatment. Eur Arch Psychiatry Clin Neurosci 264 (4), 333-343.

Gold, J.M., 2004. Cognitive deficits as treatment targets in schizophrenia. Schizophr Res 72 (1), 21-28.

Goldstein, G., 2013. Application of cluster analysis to investigate neuropsychological heterogeneity in psychiatric and neurological patients, in: Allen, D.N., Goldstein, G. (Eds.), Cluster Analysis in Neuropsychological Research. Springer, NY, USA, pp. 37-70.

Goldstein, G., Allen, D.N., Seaton, B.E., 1998. A comparison of clustering solutions for cognitive heterogeneity in schizophrenia. J Int Neuropsychol Soc 4 (4), 353-362.

Green, M.F., Nuechterlein, K.H., 2004. The MATRICS initiative: developing a consensus cognitive battery for clinical trials. Schizophr Res 72 (1), 1-3. 
Green, M.F., Nuechterlein, K.H., Gold, J.M., Barch, D.M., Cohen, J., Essock, S., et al., 2004. Approaching a consensus cognitive battery for clinical trials in schizophrenia: the NIMHMATRICS conference to select cognitive domains and test criteria. Biol Psychiatry 56 (5), 301307.

Hamilton, M., 1959. The assessment of anxiety states by rating. Br J Med Psychol 32 (1), 50-55.

Heinrichs, R.W., Miles, A.A., Ammari, N., Muharib, E., 2013. Cognitive as a central illness feature in schizophrenia, in: Harvey, P.D. (Ed.), Cognitive Impairment in Schizophrenia. Cambridge University Press, Cambridge, UK.

Heinrichs, R.W., Ruttan, L., Zakzanis, K.K., Case, D., 1997. Parsing schizophrenia with neurocognitive tests: evidence of stability and validity. Brain Cogn 35 (2), 207-224.

Hill, S.K., Ragland, J.D., Gur, R.C., Gur, R.E., 2002. Neuropsychological profiles delineate distinct profiles of schizophrenia, an interaction between memory and executive function, and uneven distribution of clinical subtypes. J Clin Exp Neuropsychol 24 (6), 765-780.

Hubert, L., Arabie, P., 1985. Comparing partitions. Journal of classification 2, 193-218.

Iasevoli, F., Giordano, S., Balletta, R., Latte, G., Formato, M.V., Prinzivalli, E., et al., 2016. Treatment resistant schizophrenia is associated with the worst community functioning among severely-ill highly-disabling psychiatric conditions and is the most relevant predictor of poorer achievements in functional milestones. Prog Neuropsychopharmacol Biol Psychiatry 65, 34-48.

Ilonen, T., Taiminen, T., Karlsson, H., Lauerma, H., Leinonen, K.M., Wallenius, E., et al., 2004. Neuropsychological subtyping of schizophrenia. Psychiatry Res 129 (2), 191-199.

Iyer, S., Jordan, G., MacDonald, K., Joober, R., Malla, A., 2015. Early intervention for psychosis: a Canadian perspective. J Nerv Ment Dis 203 (5), 356-364.

Keefe, R.S., Haig, G.M., Marder, S.R., Harvey, P.D., Dunayevich, E., Medalia, A., et al., 2016. Report on ISCTM Consensus Meeting on Clinical Assessment of Response to Treatment of Cognitive Impairment in Schizophrenia. Schizophr Bull 42 (1), 19-33.

Kendler, K.S., Ohlsson, H., Sundquist, J., Sundquist, K., 2015. IQ and schizophrenia in a Swedish national sample: their causal relationship and the interaction of IQ with genetic risk. Am J Psychiatry 172 (3), 259-265.

Khandaker, G.M., Barnett, J.H., White, I.R., Jones, P.B., 2011. A quantitative meta-analysis of population-based studies of premorbid intelligence and schizophrenia. Schizophr Res 132 (2-3), 220-227.

Kremen, W.S., Seidman, L.J., Faraone, S.V., Toomey, R., Tsuang, M.T., 2004. Heterogeneity of schizophrenia: a study of individual neuropsychological profiles. Schizophr Res 71 (2-3), 307321.

Lee, D.Y., Smith, G.N., Su, W., Honer, W.G., Macewan, G.W., Lapointe, J.S., et al., 2012. White matter tract abnormalities in first-episode psychosis. Schizophr Res 141 (1), 29-34. 
Lee, J., Rizzo, S., Altshuler, L., Glahn, D.C., Miklowitz, D.J., Sugar, C.A., et al., 2017. Deconstructing Bipolar Disorder and Schizophrenia: A cross-diagnostic cluster analysis of cognitive phenotypes. J Affect Disord 209, 71-79.

Lees, J., Applegate, E., Emsley, R., Lewis, S., Michalopoulou, P., Collier, T., et al., 2015. Calibration and cross-validation of MCCB and CogState in schizophrenia. Psychopharmacology (Berl) 232 (21-22), 3873-3882.

Lepage, M., Bodnar, M., Joober, R., Malla, A., 2015. The effect of aripiprazole on hippocampal volume in first-episode psychosis: A one year longitudinal neuro-imaging study, 12th World congress of biological psychiatry, Athens, Greece.

Lewandowski, K.E., Baker, J.T., McCarthy, J.M., Norris, L.A., Ongur, D., 2017. Reproducibility of cognitive profiles in psychosis using cluster analysis. J Int Neuropsychol Soc, 1-9.

Lewandowski, K.E., Sperry, S.H., Cohen, B.M., Ongur, D., 2014. Cognitive variability in psychotic disorders: a cross-diagnostic cluster analysis. Psychol Med 44 (15), 3239-3248.

Lindenmayer, J.P., Ozog, V.A., Khan, A., Ljuri, I., Fregenti, S., McGurk, S.R., 2017. Predictors of response to cognitive remediation in service recipients with severe mental illness. Psychiatr Rehabil J 40 (1), 61-69.

McCleery, A., Ventura, J., Kern, R.S., Subotnik, K.L., Gretchen-Doorly, D., Green, M.F., et al., 2014. Cognitive functioning in first-episode schizophrenia: MATRICS Consensus Cognitive Battery (MCCB) Profile of Impairment. Schizophr Res 157 (1-3), 33-39.

McGorry, P., Keshavan, M., Goldstone, S., Amminger, P., Allott, K., Berk, M., et al., 2014. Biomarkers and clinical staging in psychiatry. World Psychiatry 13 (3), 211-223.

Mesholam-Gately, R.I., Giuliano, A.J., Goff, K.P., Faraone, S.V., Seidman, L.J., 2009. Neurocognition in first-episode schizophrenia: a meta-analytic review. Neuropsychology 23 (3), 315-336.

Moritz, S., Andresen, B., Perro, C., Schickel, M., Krausz, M., Naber, D., et al., 2002. Neurocognitive performance in first-episode and chronic schizophrenic patients. Eur Arch Psychiatry Clin Neurosci 252 (1), 33-37.

Nuechterlein, K., McEwen, S., Ventura, J., Subotnik, K., Turner, L., Boucher, M., et al., 2018. Aerobic exercise enhances cognitive training effects in first episode schizophrenia: cognitive and functional gains and promising biological mechanisms of action. Schizophr Bull 44 (suppl_1), S18.

Nuechterlein, K.H., Barch, D.M., Gold, J.M., Goldberg, T.E., Green, M.F., Heaton, R.K., 2004. Identification of separable cognitive factors in schizophrenia. Schizophr Res 72 (1), 29-39.

Nuechterlein, K.H., Ventura, J., McEwen, S.C., Gretchen-Doorly, D., Vinogradov, S., Subotnik, K.L., 2016. Enhancing Cognitive Training Through Aerobic Exercise After a First Schizophrenia Episode: Theoretical Conception and Pilot Study. Schizophr Bull 42 Suppl 1, S44-52. 
Ohi, K., Sumiyoshi, C., Fujino, H., Yasuda, Y., Yamamori, H., Fujimoto, M., et al., 2017. A brief assessment of intelligence decline in schizophrenia as represented by the difference between current and premorbid intellectual quotient. Front Psychiatry 8, 293.

Oie, M., Sundet, K., Rund, B.R., 2010. Neurocognitive decline in early-onset schizophrenia compared with ADHD and normal controls: evidence from a 13-year follow-up study. Schizophr Bull 36 (3), 557-565.

Pietrzak, R.H., Olver, J., Norman, T., Piskulic, D., Maruff, P., Snyder, P.J., 2009. A comparison of the CogState Schizophrenia Battery and the Measurement and Treatment Research to Improve Cognition in Schizophrenia (MATRICS) Battery in assessing cognitive impairment in chronic schizophrenia. J Clin Exp Neuropsychol 31 (7), 848-859.

Pukrop, R., Schultze-Lutter, F., Ruhrmann, S., Brockhaus-Dumke, A., Tendolkar, I., Bechdolf, A., et al., 2006. Neurocognitive functioning in subjects at risk for a first episode of psychosis compared with first- and multiple-episode schizophrenia. J Clin Exp Neuropsychol 28 (8), 1388-1407.

Rehse, M., Bartolovic, M., Baum, K., Richter, D., Weisbrod, M., Roesch-Ely, D., 2016. Influence of antipsychotic and anticholinergic loads on cognitive functions in patients with schizophrenia. Schizophr Res Treatment 2016, 8213165.

Reser, M.P., Allott, K.A., Killackey, E., Farhall, J., Cotton, S.M., 2015. Exploring cognitive heterogeneity in first-episode psychosis: What cluster analysis can reveal. Psychiatry Res 229 (3), 819-827.

RStudio Team, 2015. RStudio: Integrated Development for R. RStudio, Inc., Boston, MA.

Rund, B.R., Barder, H.E., Evensen, J., Haahr, U., ten Velden Hegelstad, W., Joa, I., et al., 2016. Neurocognition and duration of psychosis: a 10-year follow-up of first-episode patients. Schizophr Bull 42 (1), 87-95.

Seaton, B.E., Allen, D.N., Goldstein, G., Kelley, M.E., van Kammen, D.P., 1999. Relations between cognitive and symptom profile heterogeneity in schizophrenia. J Nerv Ment Dis 187 (7), 414419.

Seaton, B.E., Goldstein, G., Allen, D.N., 2001. Sources of heterogeneity in schizophrenia: the role of neuropsychological functioning. Neuropsychol Rev 11 (1), 45-67.

Sponheim, S.R., Jung, R.E., Seidman, L.J., Mesholam-Gately, R.I., Manoach, D.S., O'Leary, D.S., et al., 2010. Cognitive deficits in recent-onset and chronic schizophrenia. J Psychiatr Res 44 (7), 421-428.

Szoke, A., Trandafir, A., Dupont, M.E., Meary, A., Schurhoff, F., Leboyer, M., 2008. Longitudinal studies of cognition in schizophrenia: meta-analysis. Br J Psychiatry 192 (4), 248-257.

Tabachnick, B.G., Fidell, L.S., 2013. Using Multivariate Statistics. Pearson, USA.

Twamley, E.W., Burton, C.Z., Vella, L., 2011. Compensatory cognitive training for psychosis: who benefits? Who stays in treatment? Schizophr Bull 37 Suppl 2, S55-62. 
Uren, J., Cotton, S.M., Killackey, E., Saling, M.M., Allott, K., 2017. Cognitive clusters in first-episode psychosis: overlap with healthy controls and relationship to concurrent and prospective symptoms and functioning. Neuropsychology 31 (7), 787-797.

Uttl, B., 2002. North American adult reading test: age norms, reliability, and validity. J Clin Exp Neuropsychol 24 (8), 1123-1137.

Venables, W.N., Ripley, B.D., 2002. Modern Applied Statistics with S. Fourth Edition. Springer, New York.

Weickert, T.W., Goldberg, T.E., Gold, J.M., Bigelow, L.B., Egan, M.F., Weinberger, D.R., 2000. Cognitive impairments in patients with schizophrenia displaying preserved and compromised intellect. Arch Gen Psychiatry 57 (9), 907-913.

Wilder-Willis, K., Shear, P., Steffen, J., Borkin, J., 2002. The relationship between cognitive dysfunction and coping abilities in schizophrenia. Schizophrenia Research 55, 259-267.

Yim, O., Ramdeen, K.T., 2015. Hierarchical cluster analysis: Comparison of three linkage measures and application to psychological data. The quantitative methods for psychology 11 (1), 8-21.

Zhang, T., Li, H., Stone, W.S., Woodberry, K.A., Seidman, L.J., Tang, Y., et al., 2015. Neuropsychological impairment in prodromal, first-episode, and chronic psychosis: assessing RBANS performance. PLoS ONE [Electronic Resource] 10 (5), e0125784. 